\title{
ETSI Reconfigurable Radio Systems - Software Defined Radio and Cognitive Radio Standards
}

\author{
Markus Mueck \\ Infineon Technologies AG \\ 85579 Neubiberg, Germany \\ MarkusDominik.Mueck@Infineon.com
}

\author{
Stanislav Filin, Hiroshi Harada \\ National Institute of Information and \\ Communications Technology \\ (NICT) \\ 239-0847 Yokosuka, Japan \\ \{sfilin, harada\}@,nict.go.jp
}

\author{
George Dimitrakopoulos, \\ Kostas Tsagkaris \\ University of Piraeus \\ Athens, Greece \\ \{gdimitra,ktsagk\}@unipi..gr \\ Gianmarco Baldini \\ EC - European Commission \\ 21027 Ispra (VA), Italy \\ gianmarco.baldini@j,jrc.it \\ Ari Ahtiainen \\ Nokia Research Center \\ 00045 Helsinki, Finland \\ ari.p.ahtiainen@nokia.com
}

\author{
Jens Gebert, Klaus Nolte \\ Alcatel-Lucent Deutschland AG \\ 70435 Stuttgart, Germany \\ \{J.Gebert, Klaus.Nolte\}@alcatel- \\ lucent.de \\ Jordi Pérez-Romero, Oriol \\ Sallent, Fernando Casadevall \\ Universitat Politècnica de \\ Catalunya \\ 08034 Barcelona, Spain \\ \{jorperez, sallent, ferranc\}@,tsc.upc.edu
}

\begin{abstract}
This paper details the current work status of the ETSI Reconfigurable Radio Systems (RRS) Technical Committee (TC) and gives an outlook on the future evolution. In particular, Software Defined Radio (SDR) related study results are presented with a focus on SDR architectures for Mobile Devices (MD), such as mobile phones, etc., as well as for Reconfigurable Base Stations (RBS). For MDs, a novel architecture is presented enabling the usage of SDR principles in a mass market context. Cognitive Radio (CR) principles within ETSI RRS are concentrated on two topics, a Cognitive Pilot Channel (CPC) proposal and a Functional Architecture (FA) for Management and Control of Reconfigurable Radio Systems, including Dynamic Self-Organising Planning and Management, Dynamic Spectrum Management, Joint Radio Resource Management, etc. Finally, study results are indicated which are targeting a SDR/CR security framework.
\end{abstract}

Keywords- Cognitive Pilot Channel, Cognitive Radio (CR), ETSI, Software Defined Radio (SDR)

\section{INTRODUCTION}

In the past, Software Defined Radio (SDR) and Cognitive Radio (CR) Technologies have been under joint investigation for high-end applications, such as military and public safety products, etc., since the general CR concept had emerged [1]. While CR represents the application side in the sense that a device is (partially/fully) aware of its context (radio context, application context, etc.) and dynamically adapts its parameters such that its operational objectives are reached in an optimum way; e.g., a MD is aware of surrounding Radio Access Technologies (RATs) and selects those which guarantee to fulfill its Quality of Service (QoS) requirements at the lowest cost (in terms of power consumption, etc.). SDR, on the other hand, is considered to be an "enabling technology" introducing the required level of flexibility in order to "enable" a device to adapt to its context. Then, numerous research projects, such as IST-E ${ }^{2} \mathrm{R}$ I and II [2], ICT-E $\mathrm{E}^{3}$ [3], etc., started to consider the usage of SDR/CR technology in a civil wide area (cellular) and short-range communications context on both the Reconfigurable Base Station (RBS) as well as MD side.

Most recently, these studies have triggered standardization initiatives, such as ETSI Reconfigurable Radio Systems (RRS), IEEE SCC41 [4], etc. In this framework, IEEE SCC41 is developing standards related to dynamic spectrum access networks with a focus on improved spectrum usage. Three Working Groups (WG) are currently active: IEEE P1900.4, IEEE P1900.5, and IEEE P1900.6. The IEEE 1900.4 WG has developed the IEEE standard 1900.4-2009 [4]. This standard defines a management system supporting network-terminal distributed optimization of radio resource usage and improvement in QoS in heterogeneous wireless networks. The WG is currently developing two more standards: P1900.4.1 (detailed description of interfaces and service access points defined in the IEEE standard 1900.4) and P1900.4a (enabling mobile wireless access service in white space frequency bands without any limitation on used radio interface). The IEEE 1900.5 WG is developing a draft standard which will define a set of policy languages, and their relation to policy architectures, for managing the functionality and behaviour of cognitive radios for dynamic spectrum access applications in a vendor-independent fashion. The IEEE 1900.6 WG is developing a draft standard which will define the information exchange between spectrum sensors and their clients in radio communication systems.

ETSI RRS, on the other hand, is performing work that is complementary to the IEEE SCC41 activities, as it will be detailed in the sequel of this paper. Section II presents the SDR related study results of ETSI RRS, considering the RBS and MD side independently; Section III details CR aspects focusing in particular on a Cognitive Pilot Channel (CPC) and a Functional Architecture (FA) for Management and Control of Reconfigurable Radio Systems, while Section IV highlights security issues addressed in the ETSI RRS framework. Finally, Section V gives a conclusion. 


\section{SOFTWARE DEFINED RADIO}

\section{A. Architecture of Radio Base Stations (RBS)}

ETSI RRS considers a basic architecture of RBS as illustrated by Fig. 1 [5]. The inherent requirements are commented below.

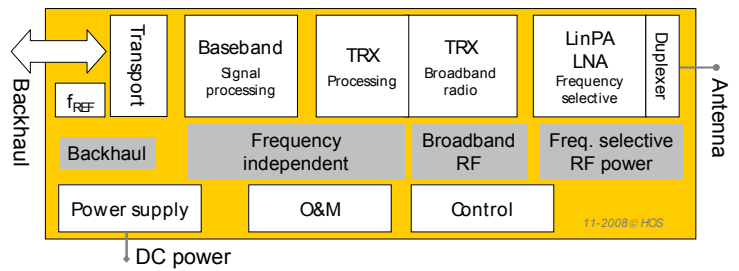

Figure 1: Basic RBS functional blocks.

\section{a) Generic requirements}

The generic requirements include, among others, the transition from one standard to another, multi-standard use, frequency re-farming, ability to participate in dynamic spectrum trading, secondary spectrum usage, dynamic capacity optimization depending on load, network planning and adaptation, antenna tuning, femtocell support, and backhaul reconfiguration.

\section{b) Operator requirements}

The operator requirements include common management of two or more systems which coexist temporally and geographically, dynamic management of hardware resources dedicated to an existing system and to a new generation system. Changes in traffic over a day, congestions in a cell, OPEX and CAPEX reductions are the main objectives in this context. This common management is also planned to be directly linked with the network planning.

\section{c) OEM requirements}

Re-configurability is considered to ease product logistics because of the expected reduction of product variants. RBS shall allow for SW upgrade of existing, as well as for capacity upgrade. Further requirements occur regarding RBS maintenance, test cases number, certification, reliability, and product roadmap management.

Based on these requirements, the following RBS architecture is proposed:

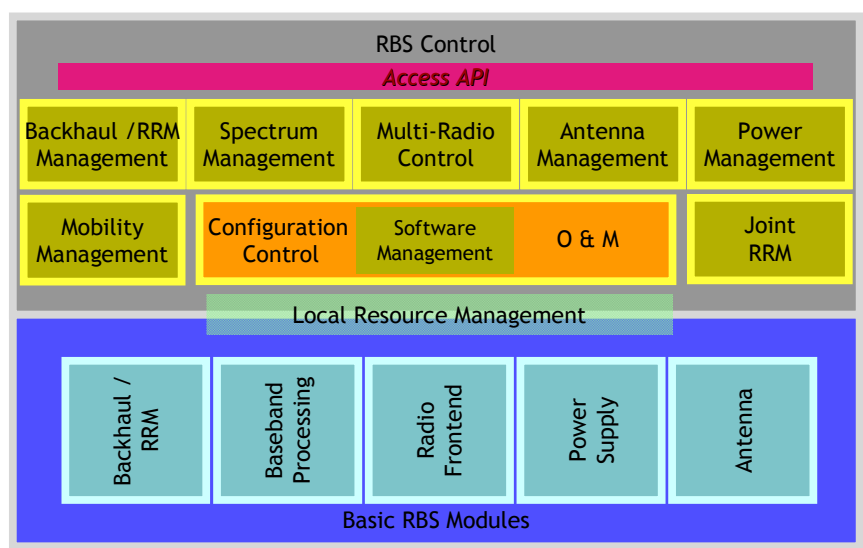

Figure 2: Reconfigurable architecture for radio base stations.
Potential candidates out of these functional blocks are considered to have potential for future standardization.

\section{B. Architecture of Mobile Devices (MDs)}

ETSI RRS has identified a set of requirements related to an SDR MD architecture [6-7], including i) general architectural requirements, ii) capability requirements, iii) operational requirements, iv) interface requirements and v) other requirements. The capability requirements are highlighted below:

a) Multiradio configuration capability: SDR equipment in mobile device is expected to install, load and activate a radio application while running a set of radio systems already.

b) Multiradio operation capability: SDR equipment in mobile device is expected to execute a number of radio systems simultaneously by taking into account temporal coexistence rules designed for their common operation.

c) Multiradio resource sharing capability: SDR equipment in mobile device is expected to execute a number of radio systems simultaneously by sharing computation, memory, communications and RF circuitry resources available on the radio computer platform by using appropriate resource allocation, binding and scheduling mechanisms.

The outcome of the study consists, among others, of the presentation of a functional architecture for SDR equipment as detailed in Fig. 3:

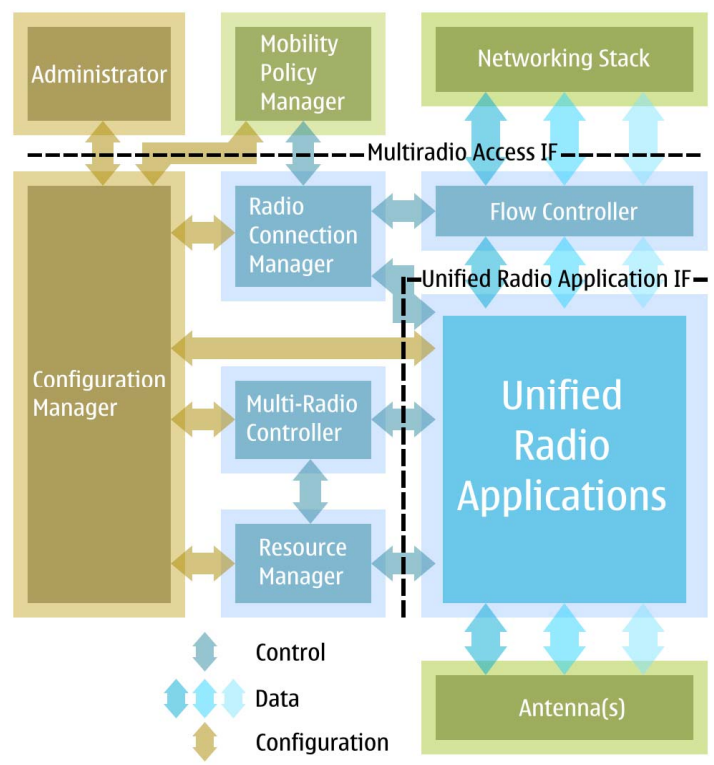

Figure 3: Functional architecture of SDR Equipment.

The components of this framework have different responsibilities as follows: 
a) Configuration Manager (CM): (de)installation and (un)loading of radio applications into radio computer as well as management of and access to the radio parameters of those radio applications.

b) Radio Connection Manager (RCM): (de)activation of radio applications according to user requests and overall management of user data flows, which can also be switched from one radio application to another.

c) Flow Controller (FC): sending and receiving of user data packets and controlling the flow.

d) Multiradio Controller (MRC): scheduling the requests on spectrum resources issued by concurrently executing radio applications in order to detect in advance the interoperability problems between them.

e) Resource Manager (RM): management of radio computer resources in order to share them among simultaneously active radio applications, while guaranteeing their real-time requirements.

Currently, ETSI RRS is focusing on the definition of the inherent interfaces (Multiradio Access Interface, Unified Radio Application Interface) as highlighted above.

\section{COGNITIVE RADIO}

In today's composite radio environment a crucial point to enable optimisation of radio resource usage is the cognitive capability of the network and terminal allowing them to switch to the most appropriate technology and frequency for the required service. This becomes even more important in a flexible spectrum management framework, where the spectrum allocated to the different RATs is foreseen to change dynamically within a range of different frequencies.

The spectrum awareness becomes a basic challenge in such generic scenario, where a number of transceivers even with flexible time-varying assignment of operating frequency and/or RAT are deployed. In this context, collaboration between network and terminals is very important. In order to provide such collaboration, the concept of a Cognitive Pilot Channel (CPC) has been developed [8-10]. As detailed in the sequel, ETSI RRS is further refining the CPC concept and presents study results related to a Functional Architecture (FA) for Management and Control of Reconfigurable Radio Systems, including Dynamic Self-Organising Planning and Management, Dynamic Spectrum Management, Joint Radio Resource Management.

\section{A. Cognitive Pilot Channel}

The CPC is defined as a channel which conveys the elements of necessary information facilitating the operations of Cognitive Radio Systems [11]. The CPC provides information on which radio accesses can be expected in a certain geographical area. This information includes operator information, radio access technology type as well as used frequencies.

Exemplary scenarios where the $\mathrm{CPC}$ is seen as useful are: a) The CPC can be used to support a terminal during the start-up phase, conveying the necessary information to let the terminal know the available RATs and corresponding used frequencies in a given geographical area.

b) In the context of a secondary system the CPC can be used to exchange sensing information between terminals and base stations in order to perform collaborative/cooperative sensing facilitating the searching of white spaces to start communication.

c) The CPC can be used for a more efficient level of collaboration between a network and the terminals by supporting Radio Resource Management (RRM) optimisation procedures and additionally for an optional dynamic spectrum access and flexible spectrum management.

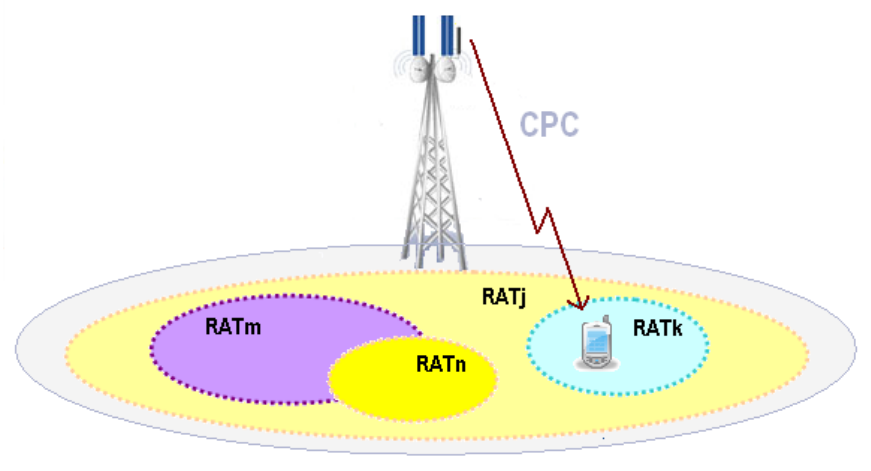

Figure 4: The CPC in a heterogeneous RAT environment.

While the basic principle of the CPC is shown in Fig. 4, different CPC solutions are possible:

In the out-band $\mathbf{C P C}$ solution, where the $\mathrm{CPC}$ is conceived as a radio channel outside the component Radio Access Technologies, the CPC either uses a new radio interface, or alternatively uses an adaptation of legacy technology with appropriate characteristics.

In the in-band CPC solution, the CPC is conceived as a logical channel within the technologies of the heterogeneous radio environment.

Combinations of out-band and in-band CPC are also possible. There, the terminal first listens on the out-band CPC in order to obtain basic parameters (e.g. available networks and their location), then the terminal selects and connects to a network, stops listening to the out-band $\mathrm{CPC}$ and starts listening on the in-band $\mathrm{CPC}$, where much more detailed context information and policies can be provided.

Further activities are ongoing for the preparation of the next World Radiocommunications conference (WRC-11), where the European Conference of Telecommunications and Postal administrations (CEPT) is investigating on the question if there is a need for a harmonized CPC frequency for the operation of Cognitive Radio Systems (CRS). One alternative is the distribution of CPC information using already assigned frequencies, e.g. as in-band CPC in existing operator networks. 
B. Functional Architecture (FA) for Management and Control of Reconfigurable Radio Systems

"Beyond third generation" (B3G) systems are aiming at the optimal joint usage of different RATs and the coordination of the available radio and spectrum resources in order to guarantee the provision and support of complex compound services, transmitted at high data rates yet still in a cost effective manner. The trend towards defining networks more flexibly and to make them adaptive (reconfigurable) to match the actual demands will help to reduce existing system inefficiencies, such as they are inherent to networks optimised for static demand patterns. Networks' interworking requires cooperation among Network Operators (NOs), so as to jointly handle extreme traffic situations, by splitting traffic among their RATs. For this purpose, all available RATs (and their spectrum and radio resources) should be accessible and usable by both the available network segments and the terminals.

Reconfigurable radio systems play a key role in this future concept, since they are able to dynamically adapt their behaviour to the varying environment requisitions by selecting most appropriate RATs and spectrum bands, etc. In this respect, this section presents, in particular, a Functional Architecture (FA) of a radio and spectrum resources optimisation platform that can be deployed in the anticipated future compound communication systems. The platform incorporates several optimisation techniques, each of which tackles a different range of application scenarios, as will be shown in the sequel.

\section{a) High level description}

The proposed FA [12] is illustrated by Fig. 5, represented as functional blocks, each of which can be considered as a wrapper to the functions deriving from the requirements mentioned above. Those blocks include: i) the Dynamic, SelfOrganising Planning and Management (DSONPM); ii) the Dynamic Spectrum Management (DSM); iii) the Joint Radio Resources Management (JRRM); and iv) the Configuration Control Module (CCM).

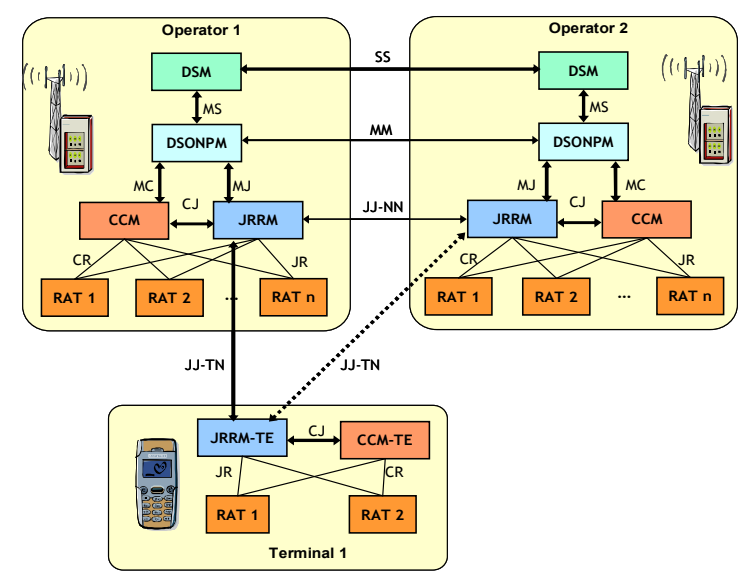

Figure 5: High level view of FA.

\section{b) Dynamic Spectrum Management (DSM)}

This clause describes the functional block that is targeted at the mid- and long-term management (e.g. in the order of hours, days) of the spectrum for the different radio systems, namely the Dynamic Spectrum Management (DSM) block. In particular, the DSM block of the FA tries to achieve an efficient utilization of the scarce and valuable spectral resources, targeted at maximizing spectrum reuse amongst users, cells, and systems, while ensuring that mutual interference between them remains at acceptable levels. The main DSM responsibilities are: i) Knowledge on the policies for the spectrum assignment, ii) Knowledge on the current spectrum assignments, iii) Provision of a spectrum framework (available amount of spectrum) to RATs, based on evaluation of spectrum occupancy and system-level parameters, iv) Knowledge on available spectrum bands for trading and v) Capability to trade available spectrum bands with other DSM instances e.g. belonging to another operator.

c) Dynamic Self-Organising Network Planning and Management (DSONPM)

The objective of the DSONPM block is to provide the medium and long term decision upon the reconfiguration actions a network segment should take, by considering certain input information, and by applying optimization functionality, enhanced with learning attributes. The decision of the optimization procedure that accompanies DSONPM is manifold and can be split as follows: i) General Application layer: QoS assignment (e.g. maximum/guaranteed bit-rate per QoS Class per cell), ii) Network layer related (distribution of traffic to RATs and networks, etc.) and iii) PHY/MAC related (RATs to be activated in the selected transceivers, Spectrum selection, etc.).

\section{d) Configuration Control Module (CCM)}

This block is mainly responsible for the execution of the reconfiguration in the overall system, following the directives provided by the other FA blocks, typically the DSONPM and the JRRM. It is needed in terminals, base stations, and other reconfigurable network elements (e.g., mobility anchors), so as to enforce and realize their adaptation to the current context, often via the CCM-RAT (CR) interface.

e) Joint management of radio resources across heterogeneous radio access technologies (JRRM)

This block is responsible for the joint management of radio resources that can belong to heterogeneous radio access technologies. Its main functionalities include: i) Access Selection, ii) Neighbourhood Information Provision for efficient discovery of available accesses in cooperation with the CPC, iii) QoS/bandwidth allocation/admission control (per user session or connection based on the requested QoS of the users' application(s)), iv) Provision of mobility and resource management directives/constraints. In particular, JRRM is distributed between the terminal and the access network. 


\section{SECURITY}

The activity of research and standardization of RRS security must resolve a broad range of issues, which spans from software assurance, conformance to spectrum regulations and certification. As a general rule, RRS must validate the communication security requirements of conventional communication systems like Data Confidentiality and Privacy, Availability, Registration, Authentication and Authorization. This is a consequence of the general conformance to standards and regulations already defined for the wireless communication systems, with which RRS must interoperate. For example, if RRS is used in the context of the public safety domain, it must support the type 1 security requirements defined in the TETRA standard. A major security issue introduced by RRS is the consequence of its reconfiguration capability (see also [13]). Theoretically RRS terminals should be able to download from the air interface new configuration profiles or software modules. Once activated, the new profile or software module will change the RRS radio transmission parameters like frequency, power and modulation types. RRS can use this capability to improve the spectrum usage efficiency and interoperability with the Radio Access Technology (RAT) present in the area. This capability presents two main security issues: i) who guarantees that the downloaded profile or software module comes from a trusted source and can be activated on the RRS terminal ? ii) who guarantees that the downloaded profile or software module will behave as expected ? One inherent requirement is that ETSI RRS needs to adopt the concept of Software Assurance in Information Technology, requiring i) a certification processes to guarantee that the software modules to be downloaded and activated will behave as expected, ii) a secure download mechanism, which guarantees the authenticity of the downloaded software. This should be completed by components in the RRS terminal to verify the software modules, iii) a secure execution environment in the RRS to guarantee that only trusted software can be activated and executed, iv) a component to ensure that spectrum regulations will be validated regardless of the software modules running on the RRS terminal.

In the military and public safety domain, there is the additional consideration that RRS can be used to remove or mitigate the interoperability barriers among the organizations involved in emergency crisis or natural disasters. This application of RRS was evaluated in the FP7 WINTSEC project [14]. Using the capability of RRS technology to communicate with different RATs using the same platform, it is theoretically possible to create interoperable "bridges" across public safety organizations, which use different communication systems. Military and public safety organizations operate with various levels of security. A significant challenge is how to design RRS wireless communication systems and terminals so that they can guarantee interoperability without sacrificing the security of data in each network.

\section{CONCLUSIONS}

This paper has illustrated the SDR and CR framework that is elaborated by ETSI RRS for a heterogeneous wide area (cellular) and short range system scenario. Key elements, such as context provision via a CPC, resource control and management by the proposed FA, a harmonized SDR architecture, security features, etc. are expected to provide the basis for a broad market acceptance. While these studies are reaching a final stage within ETSI RRS, the next step consists in elaborating corresponding normative standards. The transformation of the corresponding mandate is expected for end 2009.

\section{ACKNOWLEDGMENT}

While the contents of this paper represent the views of the authors, an acknowledgement is given to all ETSI RRS members and contributors who helped to set up this effort.

\section{REFERENCES}

[1] J. Mitola III, Cognitive Radio, Ph.D. thesis, KTH, Stockholm, Sweden, 2000

[2] FP6 E2R Programme Achievements and Impact, Dr. Didier Bourse, Dr. Markus Muck, Dr. David Bateman, Dr. Soodesh Buljore, Dr. Nancy Alonistioti, Dr. Klaus Moessner, Mr. Eric Nicollet, Dr. Enrico Buracchini, Pr. Panagiotis Demestichas, Makis Stamatelatos, Eleni Patouni, Proceeding of the SDR 07 Technical Conference and Product Exposition, available at

http://www.sdrforum.org/pages/sdr07/Proceedings/Papers/Invited/12.5001 invitedPaper1_Bourse.pdf

[3] ICT-2007-216248 E3 Project, http://www.ict-e3.eu/

[4] IEEE Standard 1900.4 for Architectural Building Blocks Enabling Network-Device Distributed Decision Making for Optimized Radio Resource Usage in Heterogeneous Wireless Access Networks, Feb. 27, 2009

[5] ETSI TR 102 681: " Reconfigurable Radio Systems (RRS)); Radio Base Station (RBS) Software Defined Radio (SDR) status, implementations and costs aspects, including future possibilities ", 2009

[6] ETSI TR 102 680: "Reconfigurable Radio Systems (RRS); SDR Reference Architecture for Mobile Device", 2009

[7] Ahtiainen, A., van Berkel, K. et al, Multiradio Scheduling and Resource Sharing on a Software Defined Radio Computing, Platform, SDR Forum Conference, Washington D.C, October 2008.

[8] Wu G., Mizuno M., and Havinga P. J. M., "MIRAI Architecture for Heterogeneous Network", IEEE Communications Magazine, Feb. 2002, vol. 40, no. 2, pp. 126- 134, Feb. 2002.

[9] IST-E ${ }^{2}$ R II Whitepaper "The E2RII Flexible Spectrum Management (FSM) Framework and Cognitive Pilot Channel (CPC) Concept Technical and Business Analysis and Recommendations"

[10] Pérez-Romero, O. Sallent, R. Agustí, L. Giupponi, "A Novel OnDemand Cognitive Pilot Channel enabling Dynamic Spectrum Allocation", DySPAN '07, 17-20 Apr., 2007

[11] ETSI TR 102 683: "Reconfigurable Radio Systems (RRS); Cognitive Pilot Channel (CPC)", 2009

[12] ETSI TR 102 682: "Reconfigurable Radio Systems (RRS); Functional Architecture for Management and Control of Reconfigurable Radio Systems", 2009

[13] Authorization and Use of Software Defined Radio: First Report and Order. Federal Communications Commission: Washington, D.C., Sept. 2001 .

[14] WINTSEC press release. http://europa.eu/rapid/pressReleasesAction.do? reference $=\mathrm{MEMO} / 06 / 375$ 